\title{
Pulmonary Function Testing and Outcomes after Left Ventricular Assist Device Implantation
}

\author{
Suraj Raheja, MD,${ }^{1}$ Hassan Nemeh, $M D,{ }^{1}$ Celeste Williams, $M D,{ }^{1}$ Cristina Tita, $M D,{ }^{1}$ Yelena Selektor, $M D,{ }^{1}$ \\ Themistokles Chamogeorgiakis, $\mathrm{MD},{ }^{1}$ David Lanfear, $\mathrm{MD}, \mathrm{MS}^{1,2}$
}

${ }^{1}$ Heart and Vascular Institute, and ${ }^{2}$ Center for Individualized and Genomic Medicine Research, Henry Ford Hospital, Detroit, Michigan, USA

\section{ABSTRACT}

Background: Pulmonary function testing (PFT) is often done during workup prior to left ventricular assist devices (LVAD), but its utility for predicting outcomes and changes in pulmonary function post-LVAD is not well established. We assessed the association of baseline PFT metrics with outcomes after LVAD, and quantified the changes in PFTs post-LVAD.

Methods and results: A retrospective study of 178 patients receiving continuous flow LVADs was conducted. A total of 129 subjects had baseline PFT data and 54 of these had repeat tests after LVAD. We collected PFT data (FEV1, FVC, FEV1/FVC ratios, and DLCO) at baseline and post-LVAD, and tested the association with survival, right heart failure, quality of life (Kansas City Cardiomyopathy Questionnaire [KCCQ]) and functional capacity (six-minute walking distance $[6 \mathrm{MWD}])$. Proportional hazards and linear regressions determined relationships between baseline PFT data and survival time and functional outcomes, respectively. Paired t-tests compared pre- and post- LVAD PFT variables. There was no association of baseline PFT parameters with survival time post-LVAD (all $P>.2$ ), nor the incidence of perioperative RV failure (all $P>.15$ ). There were no significant associations of the baseline PFT metrics with the change in KCCQ or 6MWD. There were statistically significant declines in FEV1, FEV1/FVC ratio, and DLCO after $\operatorname{LVAD}(P<.05)$.

Conclusion: In this single center study, there was no relationship between baseline PFTs and post-LVAD outcomes, and PFT parameters often worsened after LVAD. Further studies are needed to determine whether PFTs are useful in this setting, and what, if any, impact LVAD therapy has on pulmonary function.

\section{INTRODUCTION}

Systolic heart failure is one of the most prevalent disease states encountered worldwide and unfortunately there is a

Received November 15, 2018; received in revised form February 27, 2019; accepted February 28, 2019.

Correspondence: David Lanfear, Henry Ford Hospital,2799 W. Grand Blvd., Heart and Vascular Institue, Detroit, Michigan, USA; phone: (313) 917-6375 (e-mail:dlanfea1@hfbs.org). growing number of patients suffering with end-stage heart failure (HF), dictating the need for more advanced solutions [Roger 2011]. Over the past several years, left ventricular assist devices (LVADs) have taken on a greater role in clinical practice [Roberts 2015]. LVAD is an accepted treatment for end-stage $\mathrm{HF}$ patients but remains a difficult therapy to implement, is expensive, and carries significant risk of adverse outcomes such as bleeding, stroke, infection, and thrombosis [Rose 2001; Rogers 2007; Kartha 2008]. As such, patient selection is complex and challenging, but critically important.

Pulmonary function testing (PFT) is a frequently used diagnostic test in a broad spectrum of clinical settings [Olson 2013]. PFTs help to quantify pulmonary dysfunction related to chronic obstructive pulmonary disease (COPD), which is present in roughly one-third of patients undergoing cardiac surgery. COPD confers a higher risk of perioperative pulmonary and infectious complications and death [Cohen 1995]. More specifically, abnormal pulmonary function test results are associated with worse outcomes in patients undergoing cardiac surgery [Hosenpud 1990; Cohen 1995]. It seems logical that pulmonary disease may therefore impact perioperative LVAD outcomes such as right ventricular failure, the ability to be extubated, and perhaps the ultimate success of

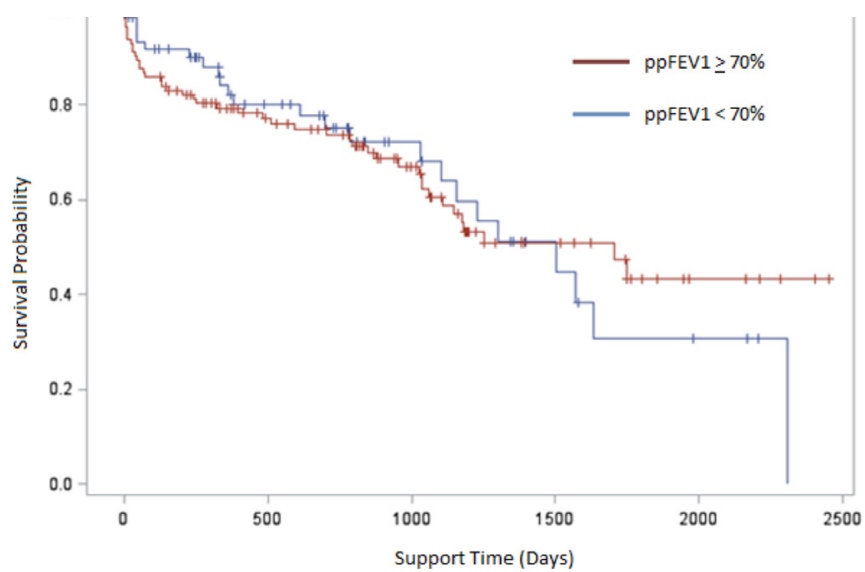

Figure 1. Survival after LVAD implantation categorized by pre-implant FEV1 ( $>70 \%$ predicted vs $<70 \%$ ). Red line is above $70 \%$ predicted and the blue line is below $70 \%$ predicted. 
Table 1. Characteristics of Patients with PFTS Prior to LVAD Implantation $(\mathrm{n}=129)$

\begin{tabular}{|c|c|}
\hline \multicolumn{2}{|l|}{ Age, y } \\
\hline Mean $( \pm S D)$ & $54.8( \pm 12.1)$ \\
\hline Range (Low to High) & 20 to 75 \\
\hline \multicolumn{2}{|l|}{ Sex, n (\%) } \\
\hline Female & $30(23)$ \\
\hline Male & $99(77)$ \\
\hline \multicolumn{2}{|l|}{ Race, n (\%) } \\
\hline White & $63(49)$ \\
\hline African American & $59(46)$ \\
\hline Asian / South Asian & $3(2)$ \\
\hline Middle Eastern & $3(2)$ \\
\hline Hispanic & $1(1)$ \\
\hline \multicolumn{2}{|c|}{ Etiology of heart failure, $\mathrm{n}(\%)$} \\
\hline Ischemic & $47(36)$ \\
\hline Non-Ischemic & $82(64)$ \\
\hline \multicolumn{2}{|l|}{ COPD diagnosis, $\mathrm{n}(\%)$} \\
\hline Yes (FEV1/FVC < 70\%) & $25(19)$ \\
\hline No (FEV1/FVC >70\%) & $104(81)$ \\
\hline \multicolumn{2}{|l|}{ Type of device, n \%) } \\
\hline HeartMate II & $114(88)$ \\
\hline Heartware & $15(12)$ \\
\hline \multicolumn{2}{|l|}{ LVAD purpose, $\mathrm{n}(\%)$} \\
\hline Destination Therapy & $68(53)$ \\
\hline Bridge to Transplant & $61(47)$ \\
\hline \multicolumn{2}{|l|}{ Support time, $d$} \\
\hline Average & 816 \\
\hline Range & 2 to 2452 \\
\hline \multicolumn{2}{|l|}{ Outcome, n (\%) } \\
\hline Alive & $49(38)$ \\
\hline Death & $49(38)$ \\
\hline Transplant & $29(22)$ \\
\hline Explant & $2(2)$ \\
\hline
\end{tabular}

therapy. On the other hand, PFTs can also be abnormal due to severe $\mathrm{HF}$ as a result of interstitial and alveolar edema, reactive fibrosis, previous pulmonary infarctions, pleural effusions, or decreased lung volumes from compressive atelectasis [Naum 1992]. Abnormalities of lung mechanics and gas transfer in heart failure have been previously linked to clinical outcomes [Kruger 2002; Lizak 2009], and it is possible that these abnormalities may also affect outcomes after LVAD. Therefore, the clinical utility of PFT in patients being considered for LVAD is an intriguing concept that nonetheless has been sparingly investigated in the current literature; to date, there are just a few descriptions of the relationship between pulmonary function and clinically relevant outcomes in the setting
Table 2. Pulmonary Function Test Data for All Patients Prior to $\operatorname{LVAD}(n=129)$

\begin{tabular}{lcc}
\hline & Average & Standard Deviation \\
\hline FEV1, L & 2.27 & 0.56 \\
PpFEV1, \% & 70.93 & 14.22 \\
FVC, L & 2.99 & 0.73 \\
PpFVC, \% & 72.50 & 13.18 \\
FEV1/FVC, \% & 76.15 & 7.93 \\
DLCO, $\mathrm{mL} / \mathrm{mmHg} / \mathrm{min}$ & 16.19 & 4.87 \\
PpDLCO, \% & 60.29 & 14.91 \\
\hline
\end{tabular}

PP indicates percent predicted, the standardized value based upon a patient's sex, age, race, and height.

Table 3. KCCQ and 6MWD Results

\begin{tabular}{lcc}
\hline & Average & Standard Deviation \\
\hline KCCQ Pre-LVAD & 52.0 & 20.3 \\
KCCQ Post-LVAD & 77.7 & 16.3 \\
KCCQ Change & 26.7 & 24.5 \\
6MWD Pre-LVAD & 214.1 & 156.6 \\
6MWD Post-LVAD & 345.3 & 87.3 \\
6MWD Change & 120.9 & 139.7 \\
\hline
\end{tabular}

KCCQ scores are the result of a 23-item questionnaire, with a scale of 0-100 (higher equals better health status). 6MWD values are reported in meters.

of LVAD [Mohamedali 2015; Arena 1999].

PFTs are included in many standard LVAD evaluation protocols, including at our center [Olson 2013]. As such, most patients progressing toward LVAD implantation have undergone testing preoperatively, and in addition many patients undergo repeat PFT after implantation, particularly those that are Bridge to Transplant (BTT) and are being reevaluated for heart transplant after LVAD [Braun 1978]. We sought to investigate the hypothesis that better PFT values would correlate to better functional and clinical outcomes after LVAD. We also set out to examine the change in PFT values after LVAD implantation.

\section{METHODS}

This study was approved by the Institutional Review Board at Henry Ford Hospital in Detroit, Michigan. Requirements for individual consent were waived due to the retrospective nature of the investigation. No funding was used to support the work, and the authors above are solely responsible for the design, conduct, analysis, and writing of this research and publication.

We performed a retrospective study of consecutive 
patients undergoing continuous flow LVAD implantation over a 7-year period from 2006 through 2013. This included 178 total patients. We extracted from the electronic health record all available PFT data. From those historical PFTs, we collected FEV1, FVC, FEV1/FVC ratios, and DLCO data, as well as the percent predicted (pp) values for each of these parameters. In total, 129 patients were eligible based on the availability of the information required above. The closest PFT prior to LVAD implantation was considered the baseline value. Among the 129 subjects with baseline PFTs available, 54 also had at least one PFT documented after LVAD implantation. Using the baseline (pre-LVAD) PFT and the most recent post-LVAD PFT available at the time of our review, we examined changes in PFT parameters after LVAD. The clinical endpoints assessed in this study included survival, incidence of perioperative right ventricular failure, and 6 month functional assessments in the forms of the Kansas City Cardiomyopathy Questionnaire [KCCQ] scoring and six-minute walking distances [6MWD].

Continuous variables are expressed as mean \pm standard deviation and categorical data as a proportion. The association of PFT parameters with survival was assessed with logrank test (dichotomized at the median) and in two proportional hazards regression models; base model (adjusted for age, race and sex) and full model (base model + indication [BTT versus DT], right ventricular function, creatinine and albumin). Model convergence was achieved in all. The association of PFT parameters with change in functional capacity (6MWD and KCCQ summary score) was tested using linear regression adjusted for baseline values. The changes in PFT parameters (FEV1, FVC, FEV1/FVC, and DLCO) from baseline to post-LVAD were compared using paired tests. $P<.05$ was considered significant. All analyses were performed in SAS 9.4 (SAS Institute, Cary, NC, USA).

\section{RESULTS}

The overall study cohort characteristics are summarized in Table 1. The cohort had a mean age of 54.8 (range 20 to $75) .23 \%$ were female (30/129), and 51\% were non-white (66/129). There were 25 patients with baseline PFT values that meet the standard definition of COPD with FEV1/FVC $<70 \%$, and of these, 4 were mild (ppFEV1 $>80 \%$ ), 16 were moderate (ppFEV1 50\% to 79\%) and 5 were severe (ppFEV1 $30 \%$ to $49 \%$ ). Destination therapy accounted for 68 of 129 patients. There was an average LVAD support time of 816 days, and there were 49 deaths during the period of study. The pulmonary function test data for these 129 patients is summarized in Table 2 . The general outcomes on quality of life and functional status as measured by KCCQ and 6MWD, respectively, are shown in Table 3.

\section{PFT Metrics and Clinical Outcomes}

Survival curves separated by those with a ppFEV1 $>70 \%$ (red) compared to patients with a ppFEV1 $<70 \%$ (blue) is shown in Figure 1. Comparing these groups by log-rank
Table 4. Cox Model Results for FEV1 \% Predicted

\begin{tabular}{lll}
\hline & Hazard Ratio & $P$ \\
\hline Base Model & & \\
Age & 0.974 & .0378 \\
African American & 0.512 & .0322 \\
Female & 1.127 & .7293 \\
FEV1 \% predicted & 1.006 & .5553 \\
Full Model & & \\
Age & 0.964 & .0099 \\
African American & 0.524 & .0385 \\
Female & 1.210 & .6050 \\
Bridge to transplant & 0.673 & .2417 \\
Right ventricular failure (preoperative) & 1.007 & .9599 \\
Creatinine & 1.497 & .2568 \\
Albumin & 1.005 & .4894 \\
FEV1 \% predicted & 1.013 & .2435
\end{tabular}

test showed no significant difference in survival probability $(P=.79)$. To test whether influenced by other baseline risk factors, we performed two sets of Cox regression models (base model and full model) for each of FEV1, ppFEV1, FEV1/ FVC or DLCO (tested one at a time) with survival time post LVAD. We found no association in any of the models tested for any of the 4 parameters (all $P>.24$, results for ppFEV1 shown in Table 4). Since pulmonary dysfunction is often associated with impaired right heart function or pulmonary hypertension, we also tested PFT parameters for association with the incidence of perioperative RV failure. Again, there was no significant association (all $P>.19$ ).

We then tested PFT parameters for association with change in functional capacity and quality of life. Among the total analysis cohort, 89 had baseline and follow-up 6MWD and 61 patients had baseline and follow-up KCCQ measurements. We tested the association of baseline PFT metrics with the change in KCCQ and 6MWD (follow up - baseline value) in linear regression models. There was no significant association of any baseline PFT metric tested (FEV1, FVC, FEV1/FVC ratio and DLCO) with either the change in KCCQ or change in $6 \mathrm{MWD}$ measures, except for ppFEV1, which showed an inverse correlation with change in $6 \mathrm{MWD}$ (ie, a lower baseline value correlated to greater 6MWD improvement).

\section{Change in PFT Metrics after LVAD Implantation}

We also sought to assess the change in PFT values from baseline to post-LVAD. A total of 54 patients had full data for analysis (ie baseline and post-LVAD PFT data). This group had a mean age of 55 (range: 20 to 75 ), was $20 \%$ female $(11 / 54)$ and $50 \%$ non-white $(27 / 54)$. There was an average of 676 days (range 108 to 1691) between the two PFTs collected for comparison. There were statistically significant declines post-LVAD implantation in almost every spirometric 
Table 5. Comparisons of Pre- And Post-LVAD PFT Parameters $(n=54)$

\begin{tabular}{lcccc}
\hline & Pre-LVAD (Average) & Post-LVAD (Average) & Change Pre to Post & T-Test Statistical Significance, $P$ \\
\hline FEV1, L & 2.25 & 2.02 & -0.23 & .0006 \\
PPFEV1, \% & 69.83 & 63.39 & -6.44 & .0012 \\
FVC, L & 2.99 & 2.84 & -0.15 & .0663 \\
PPFVC, \% & 71.87 & 69.00 & -2.87 & .1469 \\
FEV1/FVC, \% & 75.04 & 71.59 & -3.45 & .0039 \\
PPFEV1/FVC, \% & 96.54 & 91.83 & -4.71 & .0005 \\
DLCO, $\mathrm{mL} / \mathrm{mmHg} / \mathrm{min}$ & 16.89 & 14.08 & -2.81 & $<.0001$ \\
PpDLCO, \% & 62.43 & 53.12 & -9.31 & $<.0001$
\end{tabular}

parameter examined (Table 5).

To examine the clinical impact of PFT changes as well as establish clinical validity, we tested the association of changes in PFT metrics after LVAD with change in 6MWD. We found significant correlations with the change in $6 \mathrm{MWD}$ for both the change in ppFEV1 $(P=.0466)$ and change in ppDLCO $(P=.0032)$, such that a more positive change in each PFT parameter correlated with a more positive change in $6 \mathrm{MWD}$.

In order to attempt to mitigate the bias towards PFT testing in patients with more $\mathrm{SOB}$, we performed a secondary analysis restricted to the subset of patients that were bridge to transplant $(\mathrm{n}=33)$, in whom PFT testing would be done regardless of symptoms as part of pre-transplant evaluation. Even amongst these, we saw similar declines in the PFT metrics.

\section{DISCUSSION}

The data presented here shed some further light upon what has been an ambiguous area of mechanical circulatory support, pulmonary function, and LVAD outcomes. Our results suggest that preoperative PFTs add little information to pre-procedure risk stratification being poorly predictive of mid-term outcomes. Interestingly, we also found a decrement in PFT metrics post-LVAD. This finding is a bit counterintuitive and may be due to selection bias, but still deserves further careful investigation.

Our data contrasts somewhat with what limited data exists on this topic. There are a number of published abstracts [Schechter 2014], but we could find only one published manuscript on PFT and LVAD implantation [Yost 2014]. This work described the association between pulmonary function values and outcomes after LVAD placement in 103 patients and suggested worse survival in those with low ppFVC prior to LVAD. The reason for this contrast in not immediately obvious. One possible difference is that our center does exclude patients with $\mathrm{ppFEV} 1<50 \%$, so we may have been limited in the range of possible association. However, our study was larger than that one in terms of the total number of subjects. Further independent studies, preferably prospective in nature, are needed to validate our findings. If completed, this would have the clinically relevant impact of demonstrating the utility or lack of PFT in patients being considered for LVAD implantation.

Further, we noted a worsening in many PFT parameters when comparing pre-implant to post-implant values. This contrasts with a previous investigation of change in PFT measures before and after LVAD implantation, which suggested that pulmonary mechanics and gas transfer improved after device placement [Kondapaneni 2011]. However, that study was significantly limited by its study size of only three patients. Moreover, the fact that we connected the PFT changes with corresponding changes in patient functional capacity supports their internal validity. Our findings of worsening lung function should be interpreted with significant caution since follow-up PFT testing is not mandated for all patients after LVAD at our center, and so some of the tests were likely ordered due to suggestive symptoms, potentially biasing towards patients with lung disease. We tried to mitigate this concern by exploring the subgroup who were transplant candidates (who do undergo repeat PFT routinely) but the trend persisted. It is conceivable that LVAD surgery could somehow impair lung function, perhaps via impairment of diaphragm function, thoracic scarring, or chronic effusions. This is speculative, and prospective studies are needed to assess the impact of LVAD, if any, on lung function.

\section{Study Limitations}

Our study has several limitations as noted above. First, it is a single center observational experience, and it is vulnerable to the design features of a retrospective study. Second, as mentioned above, since the follow-up PFTs were clinically driven and not mandated for research, there is potential for bias in these analyses. We have tried to lessen this potential but concern remains. Finally, our cohort was systematically limited to patients with ppFEV1 $\geq 50 \%$ due to our LVAD implantation clinical practice guidelines. Thus, we cannot assess the impact of FEV values below this, and our conclusion that PFT metrics add little to risk stratification assumes PFT values meet these minimal thresholds. Additional studies in patients with severely impaired lung function at baseline would be needed to answer whether additional risk stratification is gained in these patients. 


\section{Conclusion}

In our study, preoperative PFT testing in potential LVAD patients added little information to risk stratification, and we suggest that such testing may not be routinely needed in this setting. Additionally, the clinically relevant pulmonary function measures of forced vital capacity, forced expiratory volume in one second, FEV1/FVC ratio and diffusion capacity may worsen in patients after LVAD implantation; Additional prospective investigation is needed to evaluate the impact of LVAD on lung function.

\section{REFERENCES}

Arena R, Humphrey R, McCall R. 1999. Altered exercise pulmonary function after left ventricular assist device implantation. J Cardiopulm Rehab 19:344-6.

Braun SR, Birnbaum ML, Chopra PS. 1978. Pre- and post-operative pulmonary function abnormalities in coronary artery revascularization surgery. Chest 73:316-20.

Cohen A, Katz M, Katz R, Hauptman E, Schaschner A. 1995. Chronic obstructive pulmonary disease in patients undergoing coronary artery bypass grafting. J Thoracic Cardiovasc Surg 109:574-81.

Hosenpud JD, Stibolt TA, Atwal K, Shelley D. 1990. Abnormal pulmonary function specifically related to congestive heart failure: comparison of patients before and after cardiac transplantation. Am J Med 88:493-6.

Kartha V, Gomez W, Wu B, et al. 2008. Laparoscopic cholecystectomy in a patient with an implantable left ventricular assist device. Brit J Anesthesiol 100:652-5.

Kondapaneni M, Sadanala U, Slaughter M, Perez R. 2011. Pulmonary mechanics and gas transfer after VAD implantation: Pilot study of pulmonary function test measures before and after LVAD implantation. Chest 140:889A.

Kruger S, Hoffman R, Skobel E, Breuer C, Janssens U, Hanrath P. 2002. Impairment of ventilator parameters and exercise capacity in patients with pulmonary hypertension and chronic heart insufficiency. Dtsch Med Wochenschr 127:839-44.

Lizak MK, Zakliczynski M, Jarosz A, Zembala M. 2009. The influence of chronic heart failure on pulmonary function tests in patients undergoing orthotopic heart transplantation. Transplant Proc 41:3194-7.

Mohamedali B, Bhat G, Yost G, Tatooles A. 2015. Change in spirometry after left ventricular assist device implantation. Artificial Organs 39:1046-68.

Naum CC, Sciurba FC, Rogers RM. 1992. Pulmonary function abnormalities in chronic severe cardiomyopathy preceding cardiac transplantation. Am Rev Respir Dis 145:1334-8.

Olson TP, Denzer DL, Sinnett WL, et al. 2013. Prognostic value of resting pulmonary function in heart failure. Clin Med Insights Circ Respir Pulm Med 7:35-43.

Roberts SM, Hovord DG, Kodavatiganti R, et al. 2015. Ventricular assist devices and non-cardiac surgery. BMC Anesthesiology 15:185.

Roger VL, Go AS, Lloyd-Jones DM, et al. 2011. Heart disease and stroke statistics - 2011 update: a report from the American Heart Association. Circulation 123:e18-e209.

Rogers JG, Butler J, Lansman SL, et al. 2007. Chronic mechanical circulatory support for inotrope-dependent heart failure patients who are not transplant candidates: results of the INTrEPID Trial. JACC 50:741-7.

Rose EA, Gelijns AC, Moskowitz AJ, et al. 2001. Long term use of a left ventricular assist device for end-stage heart failure. NEJM 345:1433-5.

Schechter MA, Castleberry AW, Kuchibhatla M, et al. 2014. The utility of pulmonary function tests in predicting pulmonary outcomes following destination therapy left ventricular assist device placement. J Heart Lung Transplantation 33:4S60.

Yost G, Mohamedali B, Jandura D, Sayer G, Bhat G. 2014. Pulmonary function is associated with length of stay and survival in patients receiving permanent left ventricular assist devices. J Cardiac Failure 20:8S54. 